\title{
Sensitivitätssteigerung im Rahmen eines inversen Ansatzes zur Materialparameterbestimmung für Piezokeramiken durch Elektrodenmodifikationen
}

\author{
Carsten Unverzagt, Bernd Henning \\ Universität Paderborn, EIM-E - Elektrische Messtechnik, \\ Warburger Straße 100, 33098 Paderborn \\ Unverzagt@emt.upb.de
}

\section{Kurzfassung}

Simulationen mittels der Finiten Element Methode (FEM) sind heute ein fester Bestandteil im Entwicklungsprozess von Ultraschallsystemen. Durch die simulative Ermittlung der transienten Schwingungsvorgänge können Optimierungen bezüglich gewünschter Zielkriterien vorgenommen werden. Hierdurch lassen sich die aufwendige Prototypenfertigung reduzieren und günstige Entwurfspunkte schneller ermitteln.

In vielen Ultraschallwandlern bildet eine Piezokeramik das aktive Element. Für eine realitätsnahe Simulation ist es notwendig, dass die Materialparameter dieser Piezokeramik zur Beschreibung des Materialverhaltens präzise bekannt sind. $\mathrm{Zu}$ deren Bestimmung werden verschiedene Verfahren eingesetzt, die sich in analytische und numerische Verfahren unterteilen lassen. Bei den analytischen Verfahren werden durch Vorgabe geometrischer Randbedingungen bestimmte Schwingungsformen separiert, für die sich analytische Lösungen angeben lassen [1]. Um einen vollständigen Satz an Materialparametern zu ermitteln, sind deshalb verschiedene Probekörper mit unterschiedlichen Geometrien erforderlich, deren unterschiedliche Konditionierung die Ermittlung eines konsistenten Datensatzes erschwert. Deshalb werden heute vermehrt numerische Verfahren eingesetzt, die mittels eines inversen Ansatzes eine Bestimmung der Materialparameter ermöglichen. Hierbei wird ein simulierter an einen gemessenen Impedanzverlauf durch Variationen der Materialparameter im Rahmen einer Optimierung angeglichen [2]. Einige der zu bestimmenden Materialparameter haben 
jedoch nur einen sehr geringen Einfluss auf den simulierten Impedanzverlauf und weisen deshalb nach Abschluss der Optimierung eine hohe Unsicherheit auf.

Durch eine Modifikation der Elektroden zusammen mit einem angepassten elektrischen Vornetzwerk wird hier eine Reduzierung der Symmetrie erreicht. Auf diese Weise wird die Sensitivität des Impedanzverlaufes auf die üblicherweise schwierig zu bestimmenden Materialparameter erhöht und die Ermittlung eines konsistenten Materialparametersatzes ermöglicht [3].

\section{Einleitung}

Im Folgenden werden Piezokeramiken aus dem häufig eingesetzten Material PZT (Blei-Zirkonat-Titanat) betrachtet. Das Materialverhalten wird üblicherweise mittels eines thermodynamischen makroskopischen Ansatzes beschrieben. Die sogenannten linearen Zustandsgleichungen verknüpfen die elektrischen Größen Feldstärke $\boldsymbol{E}$ und Flussdichte $\boldsymbol{D}$ über Materialtensoren mit den mechanischen Größen Spannung $\boldsymbol{T}$ und Dehnung $\boldsymbol{S}$. Im allgemeinen Fall hat beispielsweise der Tensor der Steifigkeiten [c] 81 Elemente, jedoch führen Symmetrieeigenschaften (Kräfte, Momente, Kristallklasse $6 \mathrm{~mm}$ ) und weitere thermodynamische Betrachtungen zu einer Reduktion auf zehn unabhängige Größen in den linearen Zustandsgleichungen. Mit Hilfe der Einsteinschen Summenkonvention kann hierbei die Tensorschreibweise in die übliche Matrixschreibweise aus Gleichung (1) überführt werden. Hierbei ist $\boldsymbol{e}$ die Matrix der piezoelektrischen Moduln und $\varepsilon$ die Permittivitätsmatrix.

$$
\begin{aligned}
& \boldsymbol{T}=-\boldsymbol{e}^{\mathrm{t}} \cdot \boldsymbol{E}+\boldsymbol{c}^{\mathrm{E}} \cdot \boldsymbol{S} \\
& \boldsymbol{D}=\boldsymbol{\varepsilon}^{\mathrm{S}} \cdot \boldsymbol{E}+\boldsymbol{e} \cdot \boldsymbol{S}
\end{aligned}
$$

Für eine Simulation sind neben diesen Größen zusätzlich die Dichte des Materials $\rho$ sowie Parameter für die Dämpfung erforderlich. Für transiente Simulationen wird üblicherweise das sogenannte Rayleigh-Dämpfungsmodell eingesetzt, bei dem der Dämpfungsverlauf über der Frequenz durch zwei Rayleigh-Dämpfungsparameter $\alpha_{\mathrm{M}}$ (masseproportional) und $\alpha_{\mathrm{K}}$ (steifigkeitsproportional) beschrieben wird. 
Für die FEM-Simulation wird ein rotationssymmetrisches Modell einer Piezokeramikscheibe mit Ringelektroden genutzt. Die variablen Abmessungen sind in Bild 1 dargestellt. Es kann gezeigt werden [4], dass alle erforderlichen Materialparameter auch bei der Reduzierung auf den zweidimensionalen rotationssymmetrischen Fall in den Zustandsgleichungen enthalten bleiben. Dieser ist deshalb prinzipiell zur Ermittlung der Materialparameter im Rahmen eines inversen Ansatzes geeignet.

Um während der Optimierung der Elektroden eine hohe Anzahl an Simulationen durchführen zu können, muss hier eine effiziente Methode zur Simulation des Impedanzverlaufes gewählt werden. Es bietet sich hierfür die systemtheoretische Ermittlung über eine Impulsantwort an, bei der ein diskreter Ladungsimpuls als Eingangsfunktion gewählt und die transiente Antwort ausgewertet wird.

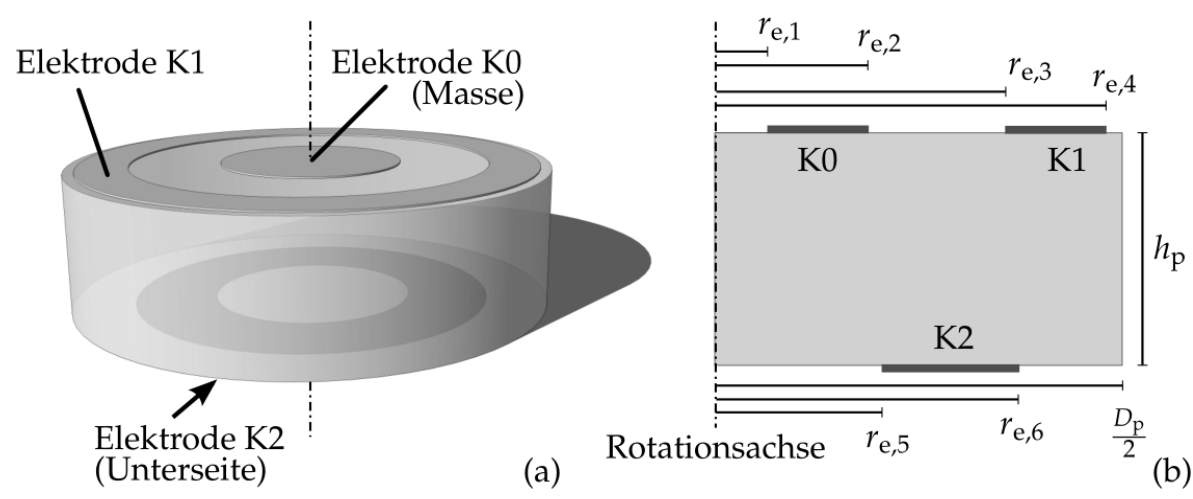

Bild 1: (a) Beispielanordnung dreier Elektroden in der dreidimensionalen Ansicht (mit $r_{\mathrm{e}, 1}=0$ ) und (b) der Kennzeichnung aller geometrischen Abmessungen.

Um sowohl die Dämpfung als auch die örtliche Diskretisierung für die FEMSimulation frequenzabhängig anpassen zu können, sind jedoch zeitaufwendige harmonische Simulationen für einzelne Frequenzstützstellen nötig. Ein Vergleich der Ergebnisse dieser beiden Verfahren zeigt, dass die Ermittlung der Impedanz mittels einer Impulsantwort für einen eingeschränkten Frequenzbereich jedoch gut als erste Näherung geeignet ist. Dieses Verfahren wird deshalb für die Optimierung der Elektrodentopologie eingesetzt. 


\section{Sensitivitätsbetrachtung}



Bild 2: Exemplarische Darstellung des Einflusses von Parametervariationen auf den Betrag des Impedanzverlaufes am Beispiel des Elastizitätsmoduls $c_{33}^{\mathrm{E}}$.

Der unterschiedliche Einfluss der einzelnen Materialparameter auf den frequenzabhängigen Impedanzverlauf kann mit Hilfe der FEM-Simulation untersucht werden. Hierfür wird zunächst eine Piezokeramikscheibe mit vollflächigen Elektroden betrachtet und jeweils ein Materialparameter variiert und der zugehörige Impedanzverlauf simuliert. Werden die Beträge der Impedanzen in Abhängigkeit von der Frequenz und der Parametervariation aufgetragen, ergibt sich die Darstellung in Bild 2.

Tabelle 1: Sensitivität des Impedanzverlaufes auf Variation einzelner Materialparameter für verschiedene Verhältnisse von $D_{\mathrm{p}} / h_{\mathrm{p}}$ im Radial- (oben) und Dickenresonanzbereich (unten). (,,+“ kennzeichnet einen deutlichen, ,-,“ einen geringen und „,“ einen nicht sichtbaren Einfluss.)

\begin{tabular}{|c|c|c|c|c|c|c|c|c|c|c|c|c|}
\hline$D_{\mathrm{p}} / h_{\mathrm{p}}$ & $c_{11}^{\mathrm{E}}$ & $c_{12}^{\mathrm{E}}$ & $c_{13}^{\mathrm{E}}$ & $c_{33}^{\mathrm{E}}$ & $c_{44}^{\mathrm{E}}$ & $e_{31}$ & $e_{33}$ & $e_{15}$ & $\varepsilon_{11}^{\mathrm{S}}$ & $\varepsilon_{33}^{\mathrm{S}}$ & $\alpha_{\mathrm{M}}$ & $\alpha_{\mathrm{K}}$ \\
\hline 3 & + & - & + & + & $\varnothing$ & - & - & $\varnothing$ & $\varnothing$ & - & - & - \\
\hline 10 & + & $\varnothing$ & + & + & $\varnothing$ & - & - & $\varnothing$ & $\varnothing$ & + & - & - \\
\hline 25 & + & $\varnothing$ & + & + & $\varnothing$ & $\varnothing$ & - & $\varnothing$ & $\varnothing$ & + & - & - \\
\hline
\end{tabular}

\begin{tabular}{|c|c|c|c|c|c|c|c|c|c|c|c|c|}
\hline$D_{\mathrm{p}} / h_{\mathrm{p}}$ & $c_{11}^{\mathrm{E}}$ & $c_{12}^{\mathrm{E}}$ & $c_{13}^{\mathrm{E}}$ & $c_{33}^{\mathrm{E}}$ & $c_{44}^{\mathrm{E}}$ & $e_{31}$ & $e_{33}$ & $e_{15}$ & $\varepsilon_{11}^{\mathrm{S}}$ & $\varepsilon_{33}^{\mathrm{S}}$ & $\alpha_{\mathrm{M}}$ & $\alpha_{\mathrm{K}}$ \\
\hline 3 & + & - & + & + & + & - & + & + & - & + & $\varnothing$ & - \\
\hline 10 & + & - & + & + & - & - & + & - & - & + & $\varnothing$ & - \\
\hline 25 & - & $\varnothing$ & - & + & - & $\varnothing$ & + & $\varnothing$ & $\varnothing$ & + & $\varnothing$ & - \\
\hline
\end{tabular}


Zusätzlich hängt der Einfluss auch von den geometrischen Abmessungen der Piezokeramik ab. Die Tabelle 1 zeigt den Einfluss für drei verschiedene Durchmesser-/Dickenverhältnisse $D_{\mathrm{p}} / h_{\mathrm{p}}$, wobei Radial- und Dickenresonanzbereich getrennt betrachtet werden. Der bereits erwähnte geringe Einfluss einiger der Materialparameter auf den Impedanzverlauf bei dieser rotationssymmetrischen Anordnung ist hier zu erkennen.

\section{Elektrodentopologie und Vornetzwerk}

Um die hohe Symmetrie der Piezokeramik zu reduzieren, wird eine Ringanordnung der Elektroden nach Bild 1 verwendet. Durch diese bleibt der Vorteil der Abbildbarkeit mittels eines zweidimensionalen FEM-Modells erhalten. Zusätzlich wird ein elektrisches Vornetzwerk verwendet. Dieses sorgt bei günstiger Dimensionierung für nicht senkrecht verlaufende Feldanteile innerhalb der Piezokeramik und erhöht auf diese Weise die Sensitivität für diejenigen Materialparameter mit bisher wenig Einfluss auf den Impedanzverlauf.

Zur Optimierung des Vornetzwerkes für ein gewünschtes Spanungsverhältnis der Elektroden K1 und K2 gegenüber K0 nach Gleichung (2) wird die Keramik als Zweitor betrachtet und die Ersatzschaltung nach Bild 3 verwendet. Die Ersatzparameter der Piezokeramik $\underline{Z}_{\mathrm{a}}, \underline{Z}_{\mathrm{b}}$ und $\underline{Z}_{\mathrm{c}}$ werden zunächst durch Simulationen verschiedener Beschaltungen der Elektroden ermittelt (Eingangs- und Ausgangskurzschlussadmittanz, Leerlauf-Kern-Impedanz). Im Anschluss erfolgt die Optimierung des Vornetzwerkes zur Einstellung des gewünschten Spannungsverhältnisses.

$\frac{1}{N} \sum_{n=1}^{N}\left(\underline{\underline{u}}_{1}\right) \geq 2 \quad$ mit $N$ : Anzahl der Werte der Spannungsvektoren

Hierbei ist $\underline{u}_{1}$ die Spannung zwischen den Elektroden K1 und K0 und $\underline{u}_{2}$ zwischen K2 und K0. Für eine Piezokeramik mit den Abmessungen $D_{\mathrm{p}}=10 \mathrm{~mm}$ und $h_{\mathrm{p}}=1 \mathrm{~mm}$ ergeben sich nach Abschluss der Optimierung die Elektrodenradien (vgl. Bild 1) zu $r_{\text {e,opt }}=\{0 ; 3,5 ; 3,8 ; 5 ; 2,05 ; 3,55\} \mathrm{mm}$ (vgl. Bild 1) mit dem Vornetzwerk aus $R_{0}=100 \Omega, \quad R_{1}=1,33 \mathrm{k} \Omega, \quad R_{2}=127 \Omega, \quad C_{1}=976 \mathrm{pF}$ und $\mathrm{C}_{2}=1 \mathrm{pF}$. 


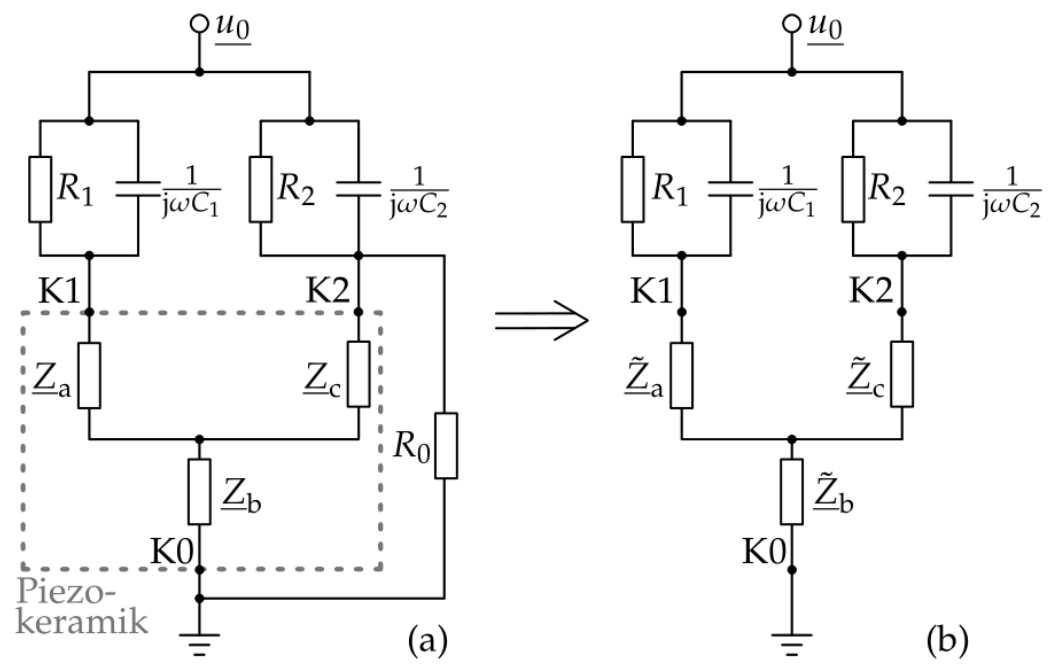

Bild 3: Das Vornetzwerk zur Annäherung eines gewünschten Spannungsverhältnisses für die Elektroden K1 und K2 (a) und die für die Netzwerkberechnung und -optimierung genutzte Ersatzschaltung (b).

\section{Angepasstes Impedanzmessverfahren}

Um den simulierten Impedanzverlauf der Piezokeramik zusammen mit dem Vornetzwerk auch messtechnisch erfassen zu können, ist ein modifizierter Messaufbau realisiert. Dieser ermöglicht die Einbindung des Vornetzwerkes und die Kontaktierung mehrerer Elektroden.

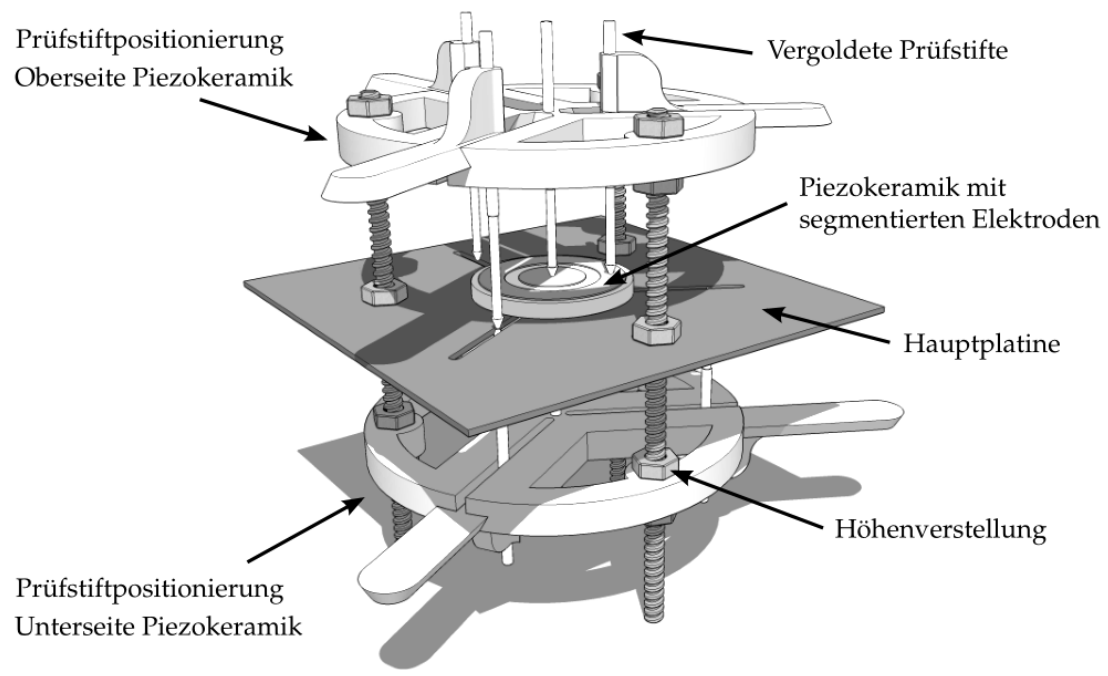

Bild 4: Schematische Darstellung des mechanischen Aufbaus zur Kontaktierung mehrerer Elektroden für die modifizierte Impedanzmessung. 


\section{Sensitivitätssteigerung}

Für das quantitative Sensitivitätsmaß $\boldsymbol{\Psi}_{\mathrm{Z}}$ nach Gleichung (3) wird eine zweidimensionale Kreuzkorrelationsfunktion ausgewertet (vgl. Bild 5). Neben den Verschiebungen in $f$ - und $Z$-Richtung $(\Delta \chi, \Delta \zeta)$ gehen dabei auch die jeweiligen Korrelationslängen $\left(\tau_{f, i}, \tau_{Z, i}\right)$ und der Wert des Maximums der Kreuzkorrelationsfunktion $\left(\kappa_{i, \max }\right)$ ein.

$$
\boldsymbol{\Psi}_{\mathrm{Z}}\left(f_{i}\right)=\frac{1}{\kappa_{i, \max }(\chi, \zeta)}\left[\left(\frac{1}{\tau_{f, i}} \cdot \Delta \chi\right)^{2}+\left(\frac{1}{\tau_{Z, i}} \Delta \zeta\right)^{2}\right]^{\frac{1}{2}}
$$

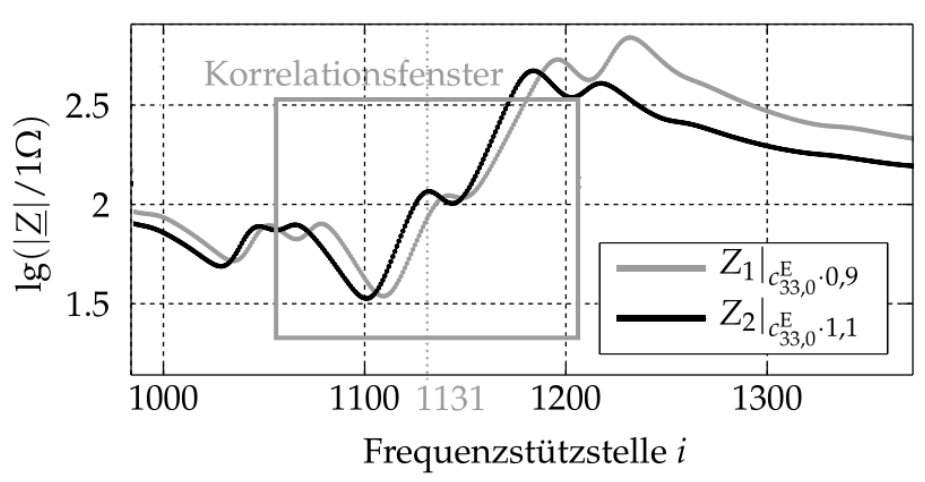

(a)

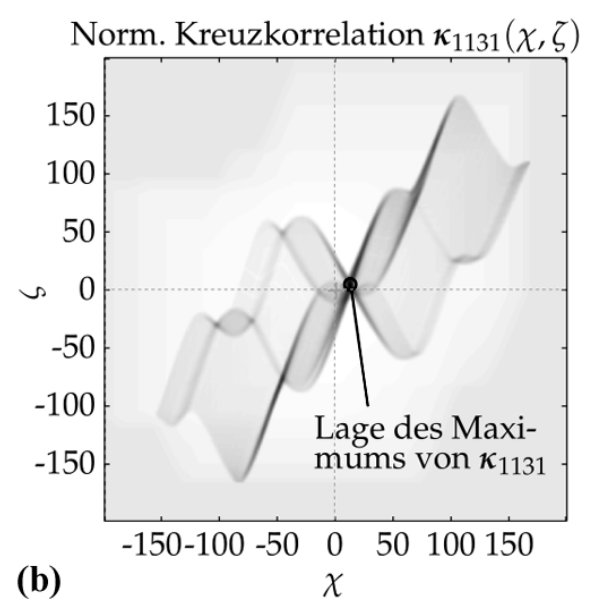

Bild 5: Ausschnitt der Impedanzverläufe (a) zur Ermittlung der normierten zweidimensionalen Kreuzkorrelationsfunktion $\kappa_{i}(\chi, \zeta)$ (b).

Dieses Sensitivitätsmaß wird für die Optimierung der Sensitivität mittels Anpassung der Elektrodenradien genutzt. Das Ergebnis der Optimierung für vier wenig sensitive Materialparameter ist in Bild 6 dargestellt. Für alle Materialparameter kann auf diese Weise ein größerer Einfluss auf den Impedanzverlauf erreicht werden. Dieser wird in Zukunft für eine Materialparameterbestimmung im Rahmen eines inversen Ansatzes genutzt werden, um die Zuverlässigkeit der bisher schwierig zu bestimmenden Materialparameter zu erhöhen. 


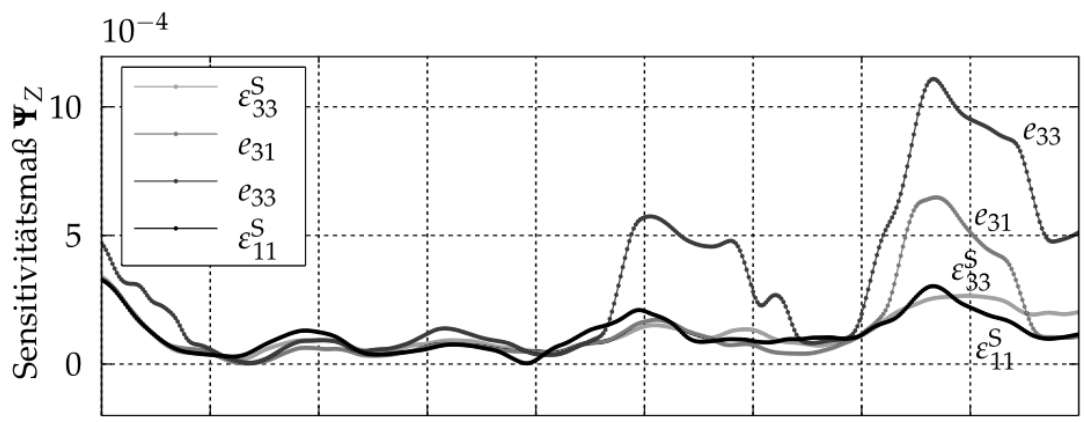

$$
10^{-4}
$$

(a)

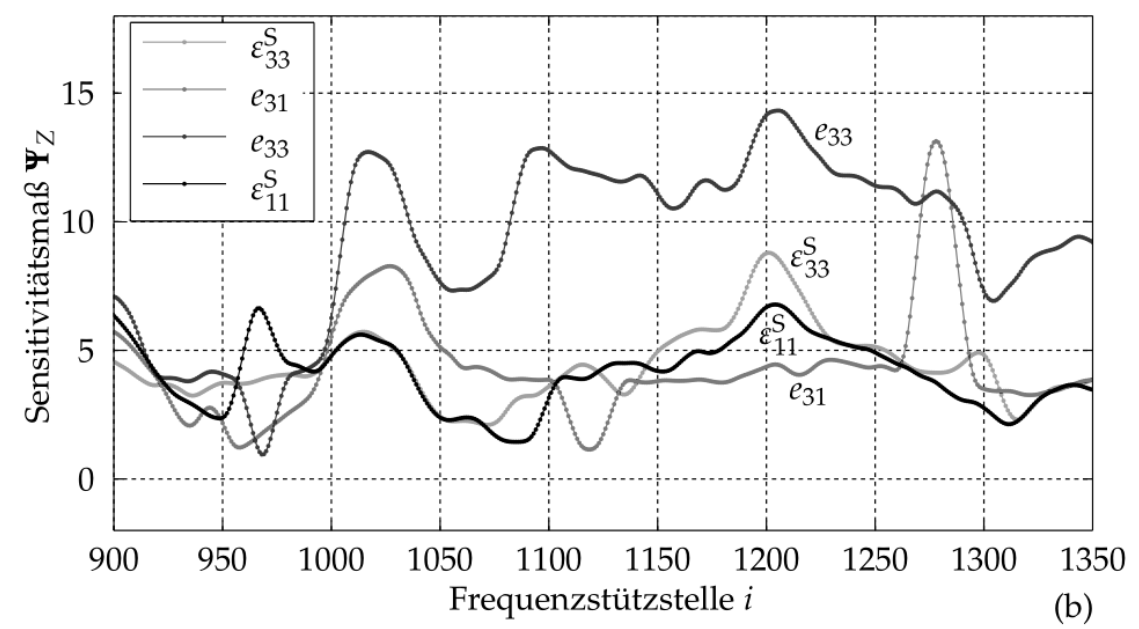

Bild 6: Sensitivitätsverläufe vor (a) und nach (b) der Optimierung der Elektrodenradien für vier Materialparameter mit kleiner Sensitivität $\left(\varepsilon_{33}^{\mathrm{S}}, e_{31}, e_{33}, \varepsilon_{11}^{\mathrm{S}}\right)$ im Bereich der Dickenresonanz der Piezokeramik.

\section{Literatur}

[1] IEEE/Ansi: 176-1987 - IEEE Standard on Piezoelectricity, 1987

[2] Kaltenbacher, B.; Lahmer, T.; Mohr, M. und Kaltenbacher, M.: PDE based determination of piezoelectric material tensors. In: European Journal of Applied Mathematics, Vol. 17, Nr. 4, S. 383-416, 2006

[3] Unverzagt, C.; Rautenberg, J.; Henning, B.und K. Kulshreshtha, K.: Modified electrode shape for the improved determination of piezoelectric material parameters. In: International Congress on Ultrasonics (ICU), Singapore, 01.-04.05.2013, Proceedings, S. 758-763

[4] Kaltenbacher, B. und Kaltenbacher, M.: Identification of Piezoelectric Material Parameters. In: Technical Report - Numerical and Symbolic Scientific Computing, SFB F013, 2008 